\title{
Resetting of Cardiac Ventricular Tachycardia Storm with Intravenous Potassium Chloride: A Case Report
}

\author{
${\text { Gal Sella }{ }^{*} \text {, Sharon L. Kracoff }}^{2}$ \\ ${ }^{1}$ Department of Cardiology, Kaplan Medical Center, Rehovot, Israel \\ ${ }^{2}$ Department of Anesthesiology, Kaplan Medical Center, Rehovot, Israel \\ Email: *galsellags@gmail.com
}

How to cite this paper: Sella, G. and Kracoff, S.L. (2018) Resetting of Cardiac Ventricular Tachycardia Storm with Intravenous Potassium Chloride: A Case Report. Case Reports in Clinical Medicine, 7, 154161.

https://doi.org/10.4236/crcm.2018.72013

Received: January 20, 2018

Accepted: February 11, 2018

Published: February 14, 2018

Copyright $\odot 2018$ by authors and Scientific Research Publishing Inc. This work is licensed under the Creative Commons Attribution International License (CC BY 4.0).

http://creativecommons.org/licenses/by/4.0/

\begin{abstract}
Ventricular Tachycardia Storm (VTS) with hemodynamic instability is a lifethreatening arrhythmia more often presents in patients with structural heart disease. Lack of response to cardioversion and/or antiarrhythmic drugs will have fatal consequences. We report a case of a hemodynamically unstable patient with Ventricular Tachycardia Storm refractory to intravenous antiarrhythmic medications, which finally responded to a slow bolus of IV potassium chloride. This case emphasizes the importance of identifying an electrolyte imbalance as a possible cause of a Ventricular Tachycardia storm and its rapid correction as an addition to the usual pharmacological treatment.
\end{abstract}

\section{Keywords}

VT Storm, Arrhythmia, Potassium, Hypokalemia

\section{Introduction}

Electrical or arrhythmic storm refers to multiple recurrences of ventricular arrhythmias over a short period of time. Ventricular tachycardia (VT) is the most frequent however; polymorphic VT and ventricular fibrillation (VF) may also result in electrical storm. The arrhythmias can be self-terminating but frequently are corrected using antiarrhythmic drugs or device-related therapies (defibrillation or anti-tachycardia pacing) [1].

Triggers for electrical storm are myocardial ischemia, acute heart failure, electrolyte disorders, hypoxia and drug-related arrhythmogenicity [2]. In particular, hypokalemia is the leading electrolyte disorder provoking an electrical storm [3] [4]. 
A Literature review revealed no reported case of resetting VTS with IV bolus of potassium chloride as a last possible resort.

We present a case of VTS that has been revoked with slow IV bolus of potassium chloride after failure of IV administration of antiarrhythmic drugs.

\section{Case Presentation}

A 61 years old Caucasian male patient with history of hyperlipidemia, overweight (BMI 32.7) and cigarette smoking (120 pack years) was admitted due to central chest pain of a crushing nature that began during sexual intercourse. Prior to admission, Electrocardiogram (ECG) demonstrated sinus rhythm, right bundle brunch block (RBBB) and ST segment elevation on leads I, aVL, V1-V6. Upon admission, physical examination revealed regular heart sounds, a systolic murmur level 2/6 with radiation to the apex and fine crepitus over the bases of the lungs. No other pathological physical findings were observed. The patient was immediately transferred to the catheterization laboratory. Coronary angiography showed complete block of the ostial left anterior descending (LAD) artery. The right coronary artery (RCA) and circumflex (Cx) showed only minor irregularities. A bare metal stent (BMS) was deployed in the proximal LAD and a drug eluting stent (DES) was deployed in the distal LAD that also showed a critical lesion. The patient was transferred to the intensive cardiac care unit (ICCU) for further monitoring and treatment.

In the ICCU, the high sensitive troponin I level reached up to $<50,000 \mathrm{pg} / \mathrm{ml}$ (normal level $<34 \mathrm{pg} / \mathrm{ml}$ ) and a potassium level of $4.6 \mathrm{mEq} / \mathrm{L}$ (normal level 3.6 $5.0 \mathrm{mEq} / \mathrm{L}$ ). On the following day, an echocardiogram demonstrated severely decreased left ventricular systolic function with an ejection fraction (EF) of $25 \%$ - 30\% and mild to moderate mitral insufficiency (Figure 1, Figure 2).

On the fourth day of hospitalization, suddenly, the cardiac monitor showed ventricular tachycardia of about 200 beats per minute (bpm) with occasional interpolated sinus rhythm lasting five to ten beats (Figure 3 ), the patient became

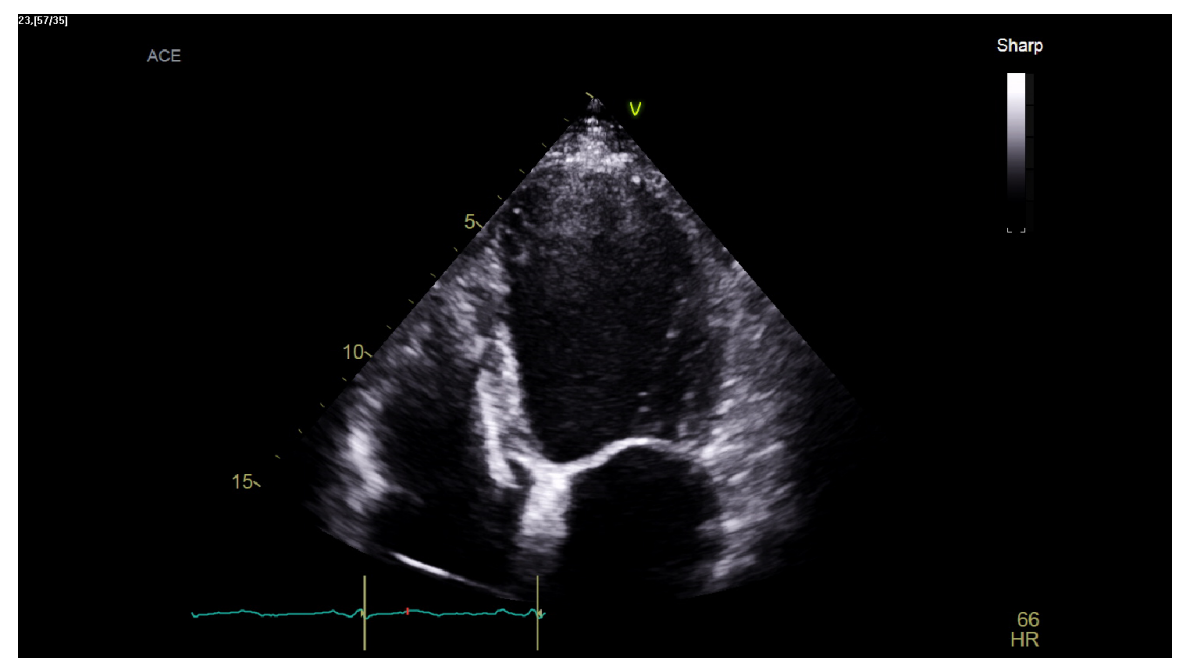

Figure 1. A four chambers view during systole. 


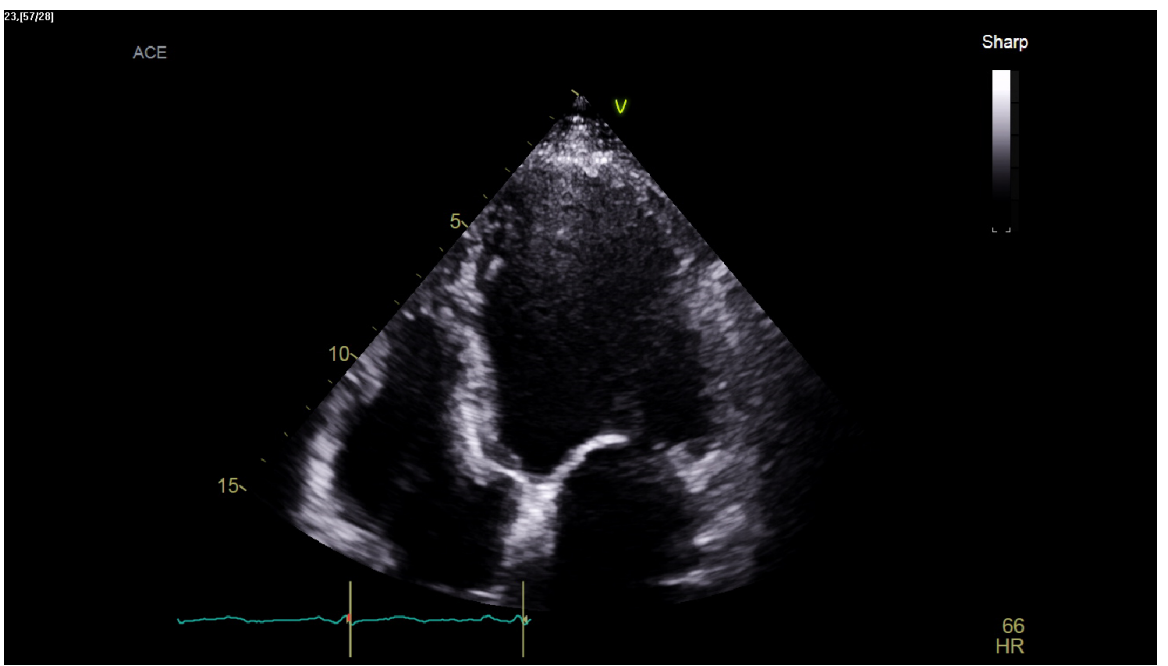

Figure 2. A four chambers view during diastole.

hemodynamically unstable, tachypneic, dyspneic, diaphoretic and unresponsive. Serum potassium level measured in the morning was $3.7 \mathrm{mEq} / \mathrm{L}$. Two boluses of $80 \mathrm{mg}$ IV Lidocaine followed by 2 boluses of $150 \mathrm{mg}$ IV Amiodarone were administered with no response (Figure 4). After several minutes of VTS, with continuous hemodynamic and consciousness deterioration and no other relatively safe therapeutic option, a syringe of $10 \mathrm{mEq}$ of Potassium chloride diluted to $10 \mathrm{ml}$ with normal saline was prepared and administered at a rate of $1 \mathrm{mEq} / \mathrm{min}$. Following administration of $5 \mathrm{mEq}$, sinus rhythm was restored (Figure 5) without any further arrhythmia, the patient regained consciousness, vital signs normalized and serum potassium level test revealed a level of $4.1 \mathrm{mEq} / \mathrm{L}$. On the following day, an Implantable Cardioverter Defibrillator (ICD) was implanted. Over the rest of the hospitalization days, the patient was hemodynamically stable, no further symptoms of acute heart failure were observed, troponin and potassium levels relatively normalized $(59 \mathrm{pg} / \mathrm{ml}$ and $4.2 \mathrm{mEq} / \mathrm{L}$ respectively). The patient discharged home in a stable condition. A month later, the patient was admitted again to the hospital due to aggravation of his heart failure. At the date of this writing, the patient is still alive.

\section{Discussion}

Pharmacologic resetting of a cardiac VT storm relies on antiarrhythmic drugssuch as beta blockers, Amiodarone and sodium channel blockers [5] and/or correction of an unbalanced ion level as in hypokalemia [5] [6]. Cardioversion is also an option, but in our case, it was considered by us as non-beneficial and even possibly detrimental, since the patient had spontaneous sporadic sinus episodes. In addition, we had to induce sedation, which could further deteriorate the patient's hemodynamic condition.

Moreover, the correction of ion imbalance is mandatory in the management of ventricular arrhythmias. The IV correction of potassium deficiency is by its administration as an intravenous solution at different concentrations. 


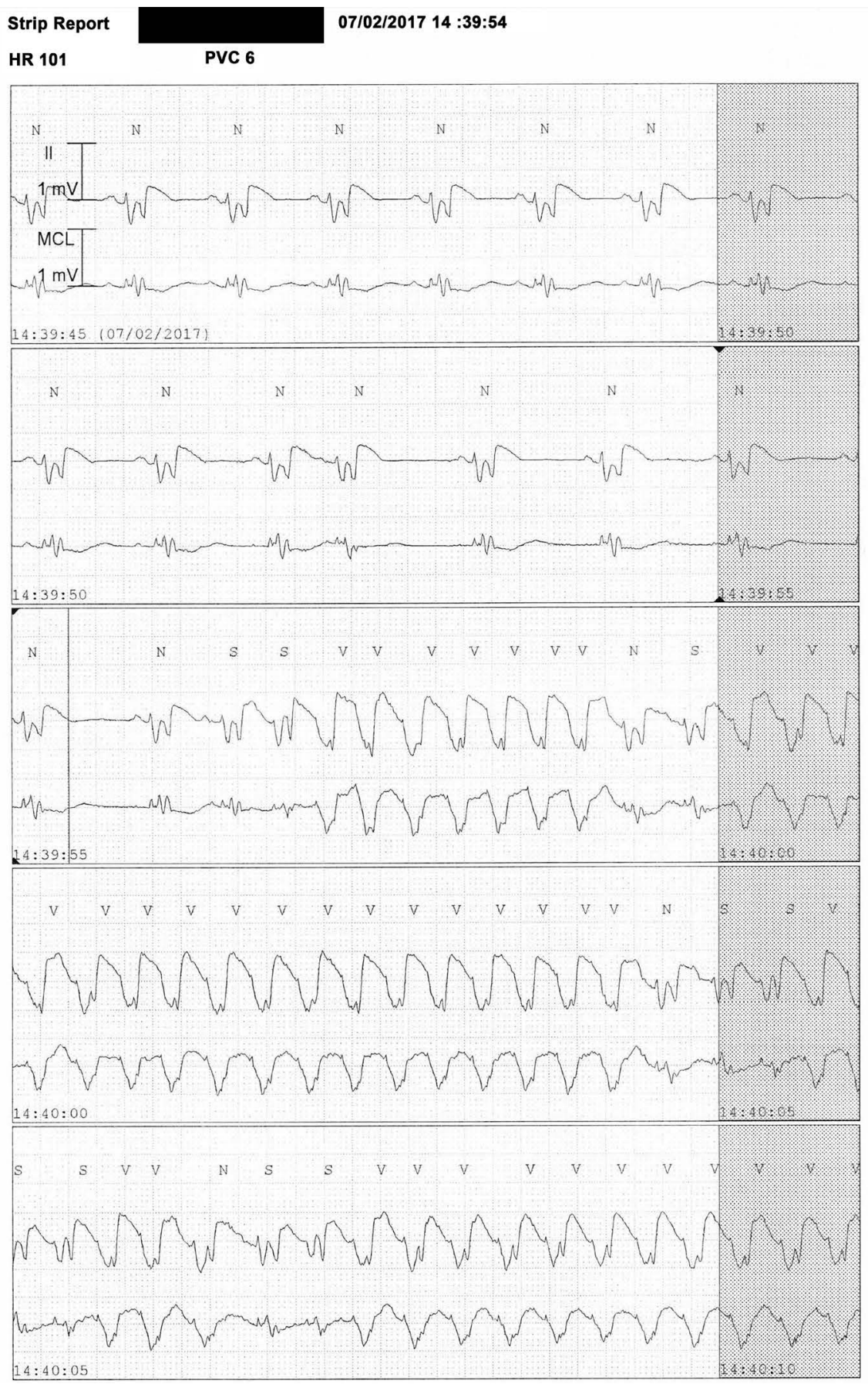

Figure 3. VT storm with sinus rhythm refractions, as observed in our patient.

Potassium chloride shouldn't be administered via IV push/bolus since uptake by the cardiomyocytes is limited by saturation of its receptors [7], and pooling of the injected potassium in the plasma can cause a transient hyperkalemia which may lead to cardiac arrest [8]. It also has an irritant and painful effect as a bolus which may cause chemical phlebitis [9].

When a patient deteriorates quickly and there is no time to infuse IV potassium chloride as the guidelines suggest [10], in spite of the well-known 


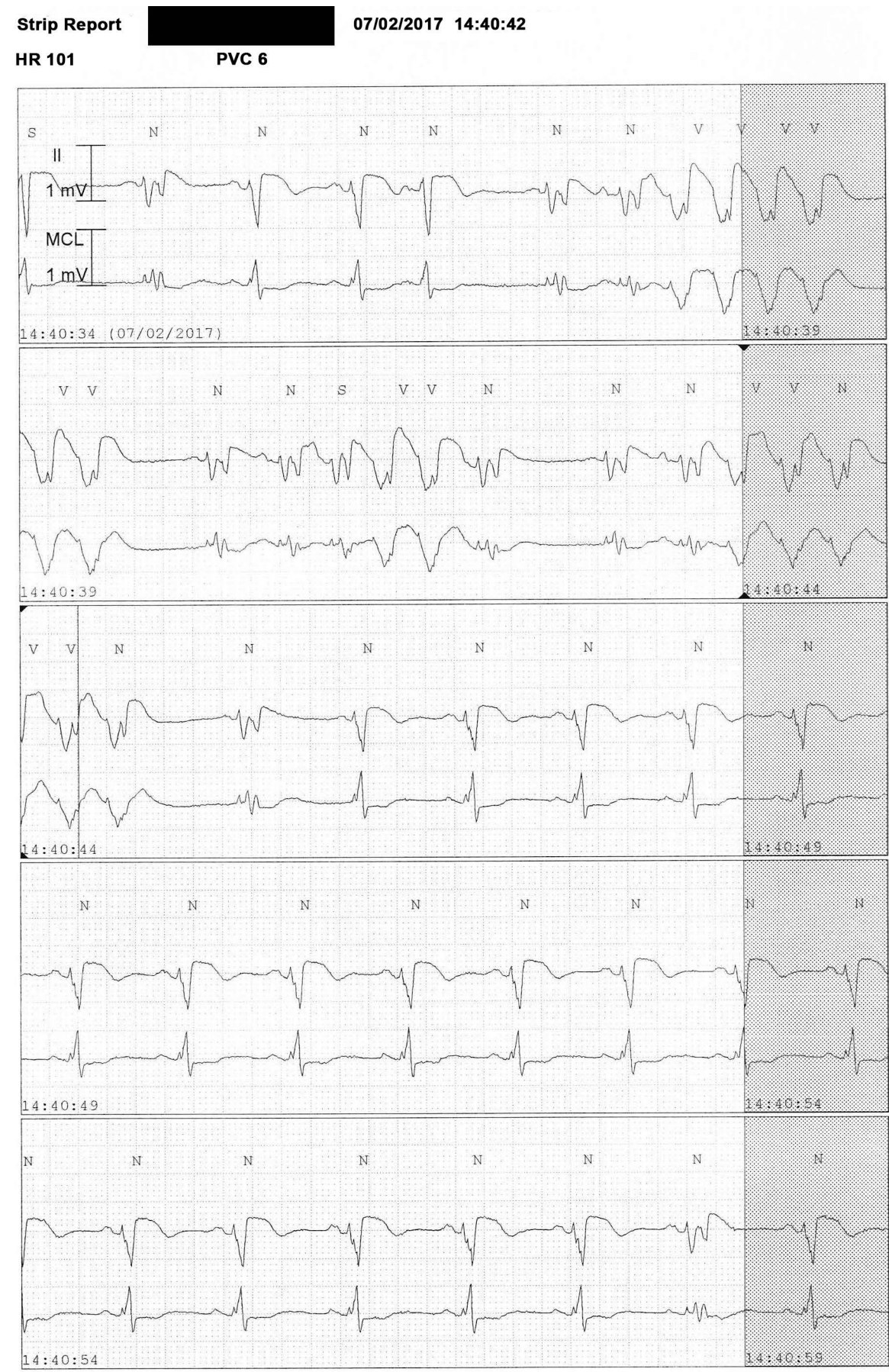

Figure 4. The VT storm remained in spite of the standard pharmacological treatment.

limitations, we believe that a bolus injection can be administered as a last lifesaving resort, as in our patient.

Hypokalemia can cause arrhythmia by interfering the resting membrane potential, by increasing threshold potential and by giving rise to sporadic automaticity. Correction of hypokalemia with potassium chloride stabilizes the cardiomyocyte membrane by causing a shift of the resting membrane potential to a less negative value and thus brings closer the threshold potential to a normal state. 
Strip Report

HR 119
07/02/2017 14:41:56

PVC 26

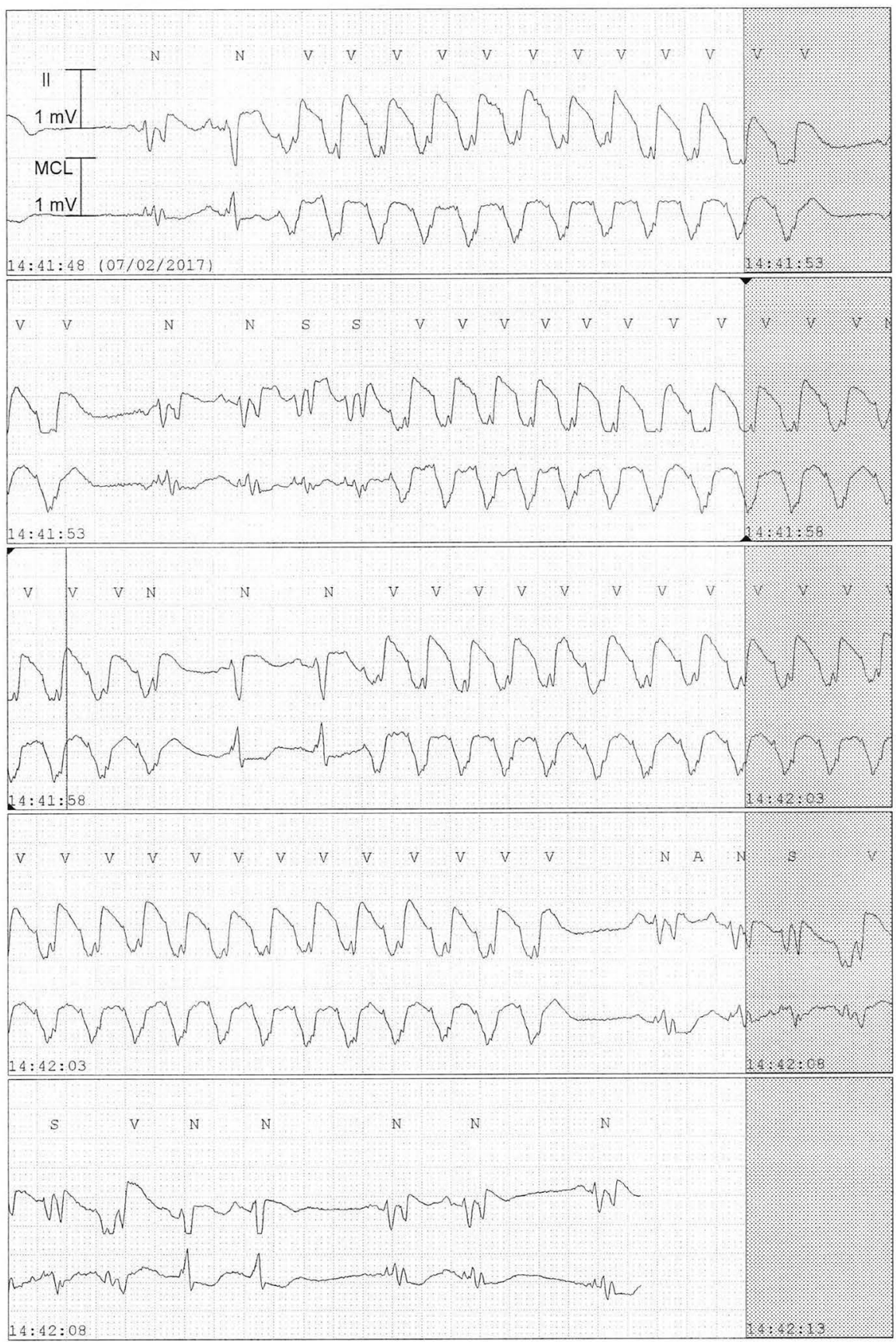

Figure 5. Resolution of the VT storm after injection of potassium chloride.

The present case report demonstrates the importance of diagnosing a hypokalemic state and treating it with potassium chloride as a part of the resolution of a VT storm.

To the best of our knowledge, this is the first reported case of resetting a VTS with potassium chloride. We speculate that the rapid correction of the hypokalemia contributed to- or caused the rhythm conversion, by itself or by means of 
facilitating the effects of the antiarrhythmic drugs. It is suggested that in life threatening arrhythmias, a rapid correction of hypokalemia can be considered as a last resort.

\section{Conclusion}

We report a case of VT storm associated with hypokalemia, which was resolved successfully with a slow bolus of IV potassium chloride. This case highlights the importance of identifying hypokalemia as a possible cause of VT storm and the implementation of an emergency correction as an addition to the usual pharmacological treatment.

\section{Learning Objectives}

1) In the setting of a VT storm, one should bear in mind that a hypokalemic state can be the trigger.

2) In life threatening arrhythmias, a rapid correction of hypokalemia can be considered as a last resort.

\section{Consent}

An informed consent was obtained from the patient.

\section{References}

[1] Rod Passman, M. (2017) Electrical Storm and Incessant Ventricular Tachycardia. Mark S Link, M., Ed.

[2] Haegeli, L.M., Della Bella, P. and Brunckhorst, C.B. (2016) Management of a Patient with Electrical Storm: Role of Epicardial Catheter Ablation. Circulation, 133, 672676. https://doi.org/10.1161/CIRCULATIONAHA.115.016336

[3] Maruyama, M., et al. (2014) Hypokalemia Promotes Late Phase 3 Early after Depolarization and Recurrent Ventricular Fibrillation during Isoproterenol Infusion in Langendorff Perfused Rabbit Ventricles. Heart Rhythm: The Official Journal of the Heart Rhythm Society, 11, 697-706. https://doi.org/10.1016/j.hrthm.2013.12.032

[4] Maruyama, M. (2014) Management of Electrical Storm: The Mechanism Matters. Journal of Arrhythmia, 30, 242-249. https://doi.org/10.1016/j.joa.2014.03.012

[5] Eifling, M., Razavi, M. and Massumi, A. (2011) The Evaluation and Management of Electrical Storm. Texas Heart Institute Journal, 38, 111-121.

[6] Almdahl, S.M., et al. (2013) Potassium-Induced Conversion of Ventricular Fibrillation after Aortic Declamping. Interactive Cardiovascular and Thoracic Surgery, 16, 143-150. https://doi.org/10.1093/icvts/ivs455

[7] Bernèche, S. and Roux, B. (2003) A Microscopic View of Ion Conduction through the K+ Channel. Proceedings of the National Academy of Sciences, 100, 8644-8648. https://doi.org/10.1073/pnas.1431750100

[8] de Oliveira, M.A.B., et al. (2014) Modes of Induced Cardiac Arrest: Hyperkalemia and Hypocalcemia-Literature Review. Revista Brasileira de Cirurgia Cardiovascular. Órgão oficial da Sociedade Brasileira de Cirurgia Cardiovascular, 29, 432-436. https://doi.org/10.5935/1678-9741.20140074

[9] Chan, R. and Armstrong, D. (2004) Peripheral Intravenous Infusion of Potassium 
Chloride: Effect of Solution Composition on Infusion-Site Pain. The Canadian Journal of Hospital Pharmacy, 57.

[10] Sorajja, D., Munger, T.M. and Shen, W.-K. (2015) Optimal Antiarrhythmic Drug Therapy for Electrical Storm. Journal of Biomedical Research, 29, 20. 